\title{
Mechanical ventilation as an indicator of somatic severity of self-poisoning: implications for psychiatric care and long-term outcomes
}

\author{
Elodie Baer, Carole Barré, Carole Fleury, Claire de Montchenu, Jean-Bernard Garré, \\ Nicolas Lerolle and Bénédicte Gohier
}

\section{Background}

Somatic severity of a self-poisoning episode varies widely between patients.

\section{Aims \\ To determine the correlates (psychiatric profiles, long-term outcome) of mechanical ventilation used as a proxy to define somatic severity during a self-poisoning.

\author{
Method \\ All patients who required mechanical ventilation were \\ pair-matched with ones who did not for age, gender \\ and presence of psychiatric history. One year after the \\ self-poisoning episode, patients were interviewed using the \\ Hospital Anxiety and Depression Scale (HADS) and a \\ quality-of-life assessment questionnaire (Short-Form 12 \\ Health Survey).
}

\section{Results}

The ventilation group $(n=99)$ more frequently had mood disorders and less frequently had adjustment disorders $(P=0.007)$, with a higher depression score on the HADS $(P=0.01)$ than those in the non-ventilation group $(n=97)$ Survival curves showed lower survival in the ventilation group $(P=0.03)$.

\section{Conclusions}

Requirement for mechanical ventilation following selfpoisoning is associated with a high prevalence of mood disorders and poor long-term outcome.

\section{Declaration of interest}

None.

\section{Copyright and usage}

(c) The Royal College of Psychiatrists 2016.
Completed suicides and suicide attempts are a major health problem, being the third cause of premature death and the first cause of death in the 15-24 year age group in high-income countries. ${ }^{1,2}$ Drug poisoning is by far the most common method, representing nearly $80 \%$ of all suicide attempts in the USA. ${ }^{3}$ In France, the number of suicide attempts is approximately 290000 per year, with 10500 deaths attributed to suicide in the national vital registry. ${ }^{4}$ In addition, the high number of unexplained deaths may indicate that the above estimation is likely far from the actual number in France. ${ }^{5}$ The characteristics of individuals who attempt suicide in high-income countries are well described in the literature, with a majority being women $(65 \%)$ in their 20 s. $^{6}$ However, suicide attempts are also associated with several characteristics that suggest further morbidity such as the presence of psychiatric disorders, particularly mood and anxiety disorders, but also the presence of ongoing somatic disease, acute social problems, addiction (alcohol and drug misuse), etc. ${ }^{6-8}$ Finally, people who self-harm have an increased risk of premature death, both from suicide attempt recurrence and from natural causes as recently established in a study performed in the UK. ${ }^{8}$ Although a few recent studies have shown that psychiatric care offered to patients with bipolar disorder improves their outcome and decreases the risk of dying by suicide, there are a lack of interventions shown to be effective after self-harm, regardless of intent. ${ }^{9}$

For intensive care unit (ICU) physicians, the somatic severity of a suicide attempt is of major importance in determining medical care, although it is generally not taken into account in subsequent psychiatric care. Indeed, in previous reports from various countries on suicide attempt recurrence prevention, this component was not taken into account. ${ }^{10-12}$ Usually, various factors are considered to predict outcome after a suicide attempt: demographic variables (age, gender, social isolation), psychiatric diagnosis (notably mood disorder, bipolar disorder, psychotic disorder, alcohol and substance misuse), psychiatric history (prior admissions to hospital) as well as psychological and suiciderelevant variables such as history of previous attempts and family history of suicide. ${ }^{713-15}$ Active precautions against discovery of a suicide attempt appear to be an important predictor of eventual suicide. ${ }^{16}$

When treating suicide attempts by drug poisoning, most patients do not require specific care in the emergency room other than administration of activated charcoal if the patient is seen within the first $2 \mathrm{~h}$ following drug ingestion or antidotes in selected cases such as $\mathrm{N}$-acetylcysteine for paracetamol overdose, and monitoring of vital signs for a few hours. In a few instances, patients develop severe acute symptoms necessitating specific supportive care. The need for endotracheal intubation and mechanical ventilation in patients with an altered mental status is by far the most common procedure required in these cases. ${ }^{17}$ As such, requirement for mechanical ventilation represents a straightforward criterion for somatic severity. In light of the above, we hypothesised that the somatic severity of a self-poisoning episode may be associated with specific psychiatric profiles and long-term outcome. The present study was designed to determine the correlates (psychiatric profiles, long-term outcome) of mechanical ventilation used as a proxy to define somatic severity during a self-poisoning episode. Specifically, we compared patients who self-poisoned who required mechanical ventilation with patients that do not require such support and who were matched for age, gender and whether or not they had received previous psychiatric care.

\section{Method}

This single-centre study was undertaken in the medical intensive care unit (MICU) of the Angers University Hospital, Angers, France after approval by the hospital ethics committee (approval 
number: 2012/22). In this hospital, all patients suspected of selfpoisoning systemically undergo initial somatic evaluation by an ICU physician in the admission section of the unit, irrespective of the $\operatorname{drug}(\mathrm{s})$ taken or presence of symptoms. Tracheal intubation and mechanical ventilation are undertaken for commonly accepted indications, namely unprotected airways, hypoventilation or profound hypoxia. In self-poisoning, these indications are typically encountered in patients with loss of consciousness. Patients not requiring ICU admission are thereafter transferred from this section. As soon as their somatic status is deemed adequate (i.e. full consciousness recovery), all patients are evaluated by the liaison psychiatric team. When there is a major risk of a recurrent attempt, whether because of an acute psychiatric state or acute suicidal ideation, transfer to a psychiatric ward takes place, if necessary without the consent of the patient. In the remaining cases, the patient can go home after arranging a prompt appointment with a psychiatrist.

\section{Patient selection and retrospective data retrieval}

Data about patients aged 18 years and older evaluated for selfpoisoning between January and December 2011 were extracted from computerised medical and psychiatric files. Patients who required mechanical ventilation (and therefore admitted to the MICU) were included in the ventilated group. These patients were pair-matched with patients admitted for self-poisoning who did not require mechanical ventilation (non-ventilation group), according to the following criteria: age in years (s.d.=5), gender and existence or not of at least one past psychiatric event (including: past or current psychiatric out-patient care or hospital admission; past or current psychotropic drug prescription). In instances where several patients who had not been ventilated could be matched to one who had been, the one with the closest date of self-poisoning to that of the patient who had been ventilated was selected. Patients with known previous self-poisoning requiring mechanical ventilation were not selected for matching.

In addition to data required for the matching process, the following parameters were retrieved from files established at the time of self-poisoning: history of suicide attempt, chronic alcohol or drug misuse, presence of debilitating chronic disease, presence of spouse or partner and children living with the patient, employment. Following psychiatric assessment performed after the self-harm episode to establish psychiatric diagnoses these were reviewed by a senior psychiatrist (B.G.) and reclassified into five categories: schizophrenia, mood disorder, adjustment disorder, personality disorder or other. Somatic parameters were also retrieved including the Simplified Acute Physiology Score II (SAPS $\mathrm{II}^{18}$ and Organ Dysfunction and Infection (ODIN) ${ }^{19}$ score calculated during the first $24 \mathrm{~h}$ after hospital admission, nadir Glasgow coma scale, suspected ingested drugs, length of mechanical ventilation, length of ICU and hospital stay, hospital survival (binary variable denoting mortality during admission). The SAPS II and ODIN scores describe the severity of acute illness in ICU patients: the SAPS II score is used to assess the disturbances in physiological parameters (i.e. ten points are attributed for blood urea nitrogen $>30 \mathrm{mmol} / \mathrm{L}),{ }^{18}$ whereas the ODIN score describes the presence of failing organs (i.e. one point is attributed for presence of haemodynamic shock). ${ }^{19}$ Given the retrospective nature of this portion of the study, the ethics committee waived the need for informed consent.

\section{Prospective data}

Six to eighteen months after the self-harm episode, patients in both groups were interviewed for a standardised assessment of their somatic and psychiatric health. First, a letter explaining the study and an option to decline to participate was sent to their home. Fifteen days later, the patient was contacted by telephone and after obtaining oral consent to participate, the following questionnaires were administered: Activities of Daily Living $(\mathrm{ADL}),{ }^{20}$ Short-Form 12 Health Survey (SF-12, version 1.0$)^{21}$ and Hospital Anxiety and Depression Scale (HADS). ${ }^{22}$ The ADL is a questionnaire designed to measure the ability to carry out six daily activities (feeding, mobility, bathing, toileting, dressing, continence), with a lower score indicating a requirement for greater assistance; ${ }^{20} \mathrm{SF}-12$ is a quality-of-life questionnaire that uses 12 questions to measure physical function and mental wellbeing from the patient's point of view; ${ }^{21}$ HADS is a 14 -item scale to determine the levels of anxiety and depression that a patient is experiencing. ${ }^{22}$ In addition, the patient was asked whether he or she had made other suicide attempt(s) since the index episode and 'Do you feel relief at having been rescued after the suicide attempt?'. Of note, the general practitioner of the patient was subsequently alerted in case of suicidal thoughts expressed during the interview, after obtaining the consent of the patient. Telephone calls for each pair of matched patients were attempted at the same delay from the self-poisoning episode. A patient was considered as unreachable after several unanswered telephone call attempts over several weeks and after verification of other telephone numbers with family members and/or attending physicians. Finally, for unreachable patients, verification as to whether the patient was alive or deceased was ascertained through interrogation of national vital registries.

\section{Statistical analyses}

Data are presented as median (interquartile range, IQR) or $n(\%)$ as appropriate. Between-group comparisons were performed using Fisher's exact test for categorical data, and unpaired or paired non-parametric tests (Mann-Whitney and Wilcoxon signed-rank tests respectively) as appropriate for continuous data. Kaplan-Meier survival curves for all-cause mortality at 600 days were constructed and compared with the log-rank test. A value of $P<0.05$ was considered statistically significant.

\section{Results}

Over the course of the study period, 884 patients (1087 episodes) were evaluated for self-poisoning in the MICU admission section of the Angers University Hospital (see online Fig. DS1 for the study flow chart). In total 99 patients (99 episodes) required mechanical ventilation (the ventilated group); of note 20 of these patients had previously had another episode of self-poisoning over the study period but without ventilation requirement. The remaining 785 patients ( 968 episodes) evaluated for self-poisoning never required mechanical ventilation. Compared with the 785 patients that were not ventilated, the 99 patients in the ventilated group were less frequently women $(43 \% v .57 \%, P=0.02)$ and were older $(44(\mathrm{IQR}=34-54)$ v. $39(\mathrm{IQR}=27-48)$ years, $P=0.02)$.

From the 785 patients that were not ventilated, 97 were matched (the non-ventilated group) with patients in the ventilated group. Two patients in the ventilated group aged more than 75 years could not be matched because of the absence of patients who were not ventilated of the same age.

\section{Data collected during the self-poisoning episode}

As a result of matching, the two groups showed no significant differences in terms of age, gender and existence of psychiatric history (Table 1). However, the ventilated group were significantly 


\begin{tabular}{|c|c|c|c|}
\hline & $\begin{array}{l}\text { Ventilated group } \\
\qquad(n=99)\end{array}$ & $\begin{array}{l}\text { Non-ventilated group } \\
\qquad(n=97)\end{array}$ & $P$ \\
\hline Age, years: median (interquartile range) & $44(35-54)$ & $44(33-53)$ & 0.39 \\
\hline Female, $n(\%)$ & $45(45)$ & $45(46)$ & 0.42 \\
\hline Psychiatric history (any of the following), $n$ (\%) & $83(84)$ & $82(85)$ & 0.94 \\
\hline Psychiatric follow-up (past or current) & $69(70)$ & $66(68)$ & 0.84 \\
\hline Past hospital admission in psychiatry & $65(66)$ & $52(54)$ & 0.10 \\
\hline Psychotropic drug treatment (past or current) & 78 (79) & $63(65)$ & 0.04 \\
\hline Past self-harm episode & $68(69)$ & $54(56)$ & 0.08 \\
\hline Spouse or partner, $n(\%)$ & $41(41)$ & $42(43)$ & 0.79 \\
\hline Children (one or more), $n$ (\%) & $62(63)$ & $60(62)$ & 0.99 \\
\hline Employment, $n$ (\%) & $33(33)$ & $26(27)$ & 0.34 \\
\hline Drug misuse (past or current), $n$ (\%) & $11(11)$ & $7(7)$ & 0.14 \\
\hline Alcohol misuse (current), $n$ (\%) & $32(32)$ & $33(34)$ & 0.22 \\
\hline Chronic debilitating disease, ${ }^{a} n(\%)$ & $23(23)$ & $10(10)$ & 0.02 \\
\hline
\end{tabular}

more frequently on psychotropic medication (past or current). The groups did not differ with regard to sociofamilial items (employment status, family structure) or for alcohol and drug misuse. The ventilated group had a higher frequency of chronic debilitating disease.

There were no differences between the two groups with regard to suspected drugs ingested, except for carbamates, which were more frequently encountered in the ventilated group. Acute ICU severity scores were significantly higher in the ventilated group, together with longer hospital stays. Two patients in the ventilated group died during their hospital stay (massive inhalation of gastric content prior to endotracheal intubation, leading to refractory hypoxia in both patients).

More importantly, the type and frequency of psychiatric diagnoses differed significantly between the two groups (Table 3). The majority of the ventilated group had mood disorders. Conversely, the non-ventilated group were less likely to have mood disorders, although nonetheless encountered in a substantial number, whereas adjustment disorders and addictions were more frequently observed in this group. Frequency of transfer to a psychiatric hospital on completion of medical care did not differ between the two groups (44 (44\%) v. 40 (40\%) respectively, $P=0.83$ )

\section{Long-term outcome}

Participants in both groups were contacted by telephone on average $13.2(\mathrm{IQR}=9-16)$ v. $13.8(\mathrm{IQR}=9-15)$ months respectively $(P=0.44)$ after the self-poisoning episode. The response rate at follow-up differed significantly between the two groups (Fig. 1), with more in the non-ventilated group being unreachable despite a number of attempts (at least six on different days). Evidence for survival or death was obtained for all unreachable patients except for one non-French patient for whom verification of the national vital registry was not relevant. In surviving unreachable patients, failure of contact was related to relocation without an available

\begin{tabular}{|c|c|c|c|}
\hline & $\begin{array}{l}\text { Ventilated group } \\
\qquad(n=99)\end{array}$ & $\begin{array}{l}\text { Non-ventilated group } \\
\qquad(n=97)\end{array}$ & $P$ \\
\hline Simplified Acute Physiology Score II, median (IQR) & $35(28-43)$ & $13(6-13)$ & $<0.001$ \\
\hline Organ Dysfunction and Infection score, median (IQR) & $2(2-2)$ & 0 & $<0.001$ \\
\hline Glasgow coma scale, median (IQR) & $6^{a}(5-8)$ & $15(12-15)$ & $<0.001$ \\
\hline \multicolumn{4}{|l|}{ Toxic agent ingested (suspected), $n$ (\%) } \\
\hline Tricyclic antidepressant & $4(4)$ & $0(0)$ & 0.12 \\
\hline Anxiolytic carbamates & $11(11)$ & $0(0)$ & 0.03 \\
\hline Lithium & $5(5)$ & $5(5)$ & 0.91 \\
\hline Opioids & $5(5)$ & $1(1)$ & 0.24 \\
\hline Paracetamol & $5(5)$ & $4(4)$ & 0.85 \\
\hline Non-cyclic antidepressant & $26(26)$ & $19(20)$ & 0.47 \\
\hline Antipsychotic & $21(21)$ & $17(18)$ & 0.72 \\
\hline Benzodiazepine & $66(67)$ & 71 (73) & 0.6 \\
\hline Alcohol & $16(16)$ & $26(27)$ & 0.19 \\
\hline Non-steroidal anti-inflammatory drug & $10(10)$ & $7(7)$ & 0.68 \\
\hline Intensive care unit admission after initial evaluation, $n$ (\%) & $99(100)$ & $15(15)$ & $<0.001$ \\
\hline Mechanical ventilation duration, days: median (IQR) & $2(1-3)$ & $0(0)$ & $<0.001$ \\
\hline Length of intensive care unit stay of admitted patients, days: median (IQR) & $3(2-4)$ & $1(1-1)$ & $<0.001$ \\
\hline Length of hospital stay, days: median (IQR) & $6(4-9)$ & $1(1-2)$ & $<0.001$ \\
\hline Hospital mortality, $n$ (\%) & $2(2)$ & $0(0)$ & 0.5 \\
\hline
\end{tabular}




\begin{tabular}{|c|c|c|c|}
\hline & $\begin{array}{l}\text { Ventilated group, } n(\%) \\
\qquad(n=99)\end{array}$ & $\begin{array}{l}\text { Non-ventilated group, } n \text { (\%) } \\
\qquad(n=97)\end{array}$ & $P$ \\
\hline Schizophrenia & $9(9)$ & $8(8)$ & 1 \\
\hline Adjustment disorders & $17(17)$ & $30(31)$ & 0.03 \\
\hline Mood disorders & $55(56)$ & $37(38)$ & 0.01 \\
\hline Personality disorders & $8(8)$ & $4(4)$ & 0.4 \\
\hline Addictions & $4(4)$ & $14(14)$ & 0.01 \\
\hline Missing data & $6(6)$ & $4(4)$ & 0.5 \\
\hline
\end{tabular}

address or social isolation (data not shown). In addition to the two patients in the ventilated group that died in the ICU, five others in this group died after hospital discharge, all of which were secondary to suicide. Of note, these five patients all had mood disorder. By contrast, two patients in the non-ventilated group died after discharge, both of natural causes. Thus, suicide mortality in the ventilated group was 7/99 v. 0/95 in the non-ventilated group $(P<0.01)$. Survival curves for all-cause mortality beginning on the admission day for the self-poisoning episode were constructed and showed lower survival in the ventilated group (Fig. 2).

The 45 matched pairs of interview respondents were compared with regard to long-term assessment (Table 4). Only four participants in the ventilated group had an ADL score $<6$ (indicating dependency for a least one daily living activity) with all of these patients having some loss of autonomy, already noted during the self-poisoning episode, associated with neurological diseases or joint disease. The ventilated group exhibited greater depressive symptoms on the HADS in comparison with the

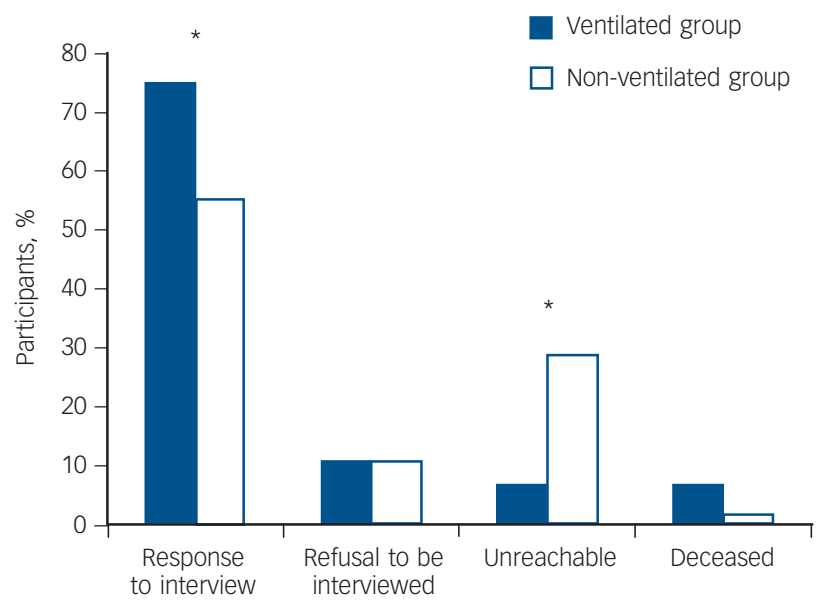

Fig. 1 Responses to telephone call attempts in the ventilated $(n=99)$ and non-ventilated $(n=97)$ group.

$P<0.001$ for overall comparison; * for $P<0.05$. non-ventilated group. No difference was observed for the anxiety score on the HADS as well as for the mental and physical components of the SF-12. Of note, when all respondents in the two groups were compared (74 in the ventilated group v. 55 in the non-ventilated group), similar results were observed (data not shown).

In response to the question 'Do you feel relief at having been rescued after the suicide attempt?' most of the patients in both groups answered 'yes' (ventilated group: yes 59 (80\%), no 2 (3\%), do not know 13 (18\%); non-ventilated group: yes 45 $(82 \%)$, no $2(4 \%)$, do not know $8(15 \%))$, with no difference between the two groups $(P=0.7)$.

\section{Discussion}

\section{Main findings}

The present study compared psychiatric characteristics and longterm outcome of patients with self-poisoning requiring mechanical ventilation with that of patients with self-poisoning who did not require mechanical ventilation and matched for age, gender and whether or not they had a psychiatric history. Requirement for

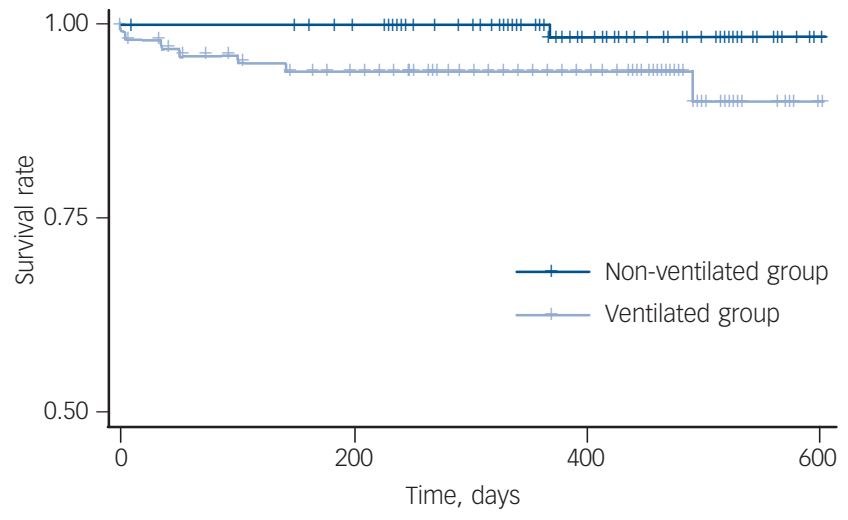

Fig. 2 Kaplan-Meier survival curves (all-cause mortality) for the ventilated $(n=99)$ and non-ventilated $(n=97)$ group $(P=0.027)$.

\begin{tabular}{|c|c|c|c|}
\hline & $\begin{array}{l}\text { Ventilated group } \\
\qquad(n=45)\end{array}$ & $\begin{array}{l}\text { Non-ventilated group } \\
\qquad(n=45)\end{array}$ & $P$ \\
\hline Activities of Daily Living <6, $n$ (\%) & $4(8)$ & $0(0)$ & 0.11 \\
\hline \multicolumn{4}{|c|}{ Short Form-12, median (interquartile range) } \\
\hline Physical component & $50.3(35-55)$ & $49.8(29-53)$ & 0.23 \\
\hline Mental component & $43.9(29-53)$ & $45.2(35-54)$ & 0.38 \\
\hline \multicolumn{4}{|c|}{ Hospital Anxiety and Depression Score, median (interquartile range) } \\
\hline Anxiety & $7(4-11)$ & $7(4-11)$ & 0.73 \\
\hline Depression & $8(2-13)$ & $5(1-8)$ & 0.02 \\
\hline
\end{tabular}


mechanical ventilation was used as a straightforward criterion for somatic severity of the suicide attempt. This simple criterion was found to delineate a specific population with very frequent mood disorders at high risk of poor long-term outcome, including vital outcome (death). Although these results should be treated with caution given the limited number of patients studied, the death rate in the ventilated group (i.e. $7 \%$ at 12 months) was strikingly well above the usually reported rate (about $1 \%$ per year) in unselected patients after a suicide attempt in Europe. ${ }^{23}$ In young patients, this represents a considerably high number of life years lost. These results confirm and extend those of Owens et al performed in the UK in which the level of consciousness alteration was associated with subsequent suicide in a 16-year outcome study, ultimately showing that the risk of suicide occurs very early after the index episode and can be assessed through a very simple criterion. $^{24}$

\section{Significance of our findings}

The observed association between somatic severity of a self-harm episode and poor long-term outcome in patients who frequently have mood disorders is consistent with findings reported in the literature. Indeed, the association between mood disorders and severity of suicidal attempt has been well described in high-income countries. ${ }^{25-27}$ In the absence of any difference in the types of ingested drugs between the two groups in our study (other than carbamates, which involved a small number of patients), somatic severity was likely related to the amount of ingested drugs rather than to the nature of the drugs, although this could not be confirmed as ingested doses were not documented. Nonetheless, this finding indirectly argues for a relationship between somatic severity of self-poisoning and the intensity of suicidal intent, on the assumption that the amount of ingested drugs is related to suicidal intentionality, which is ultimately in accordance with the observed long-term outcome. These data further corroborate and extend those of the Finnish study of Suominen et al ${ }^{28}$ whereby the intensity of suicidal intent at the time of the gesture was shown to be a risk factor for long-term mortality by suicide. The higher frequency of chronic debilitating diseases in the ventilated group is also in keeping with their psychiatric profile and outcome, as such diseases are well-known factors for mood disorders and suicide. ${ }^{29}$ Alternatively, it is possible that ICU care and the use of mechanical ventilation may have had a deleterious impact on mental health in itself. Indeed, it has been well demonstrated that an ICU stay and ICU-acquired delirium are associated with a high risk of longterm cognitive impairment, and potentially post-traumatic stress disorder, which may aggravate a previously ongoing psychiatric disease. $^{30,31}$ Unfortunately, it is impossible to determine the respective impact of psychiatric disease and of ICU care on outcome from the present data. Notwithstanding the above, our data showing altered long-term outcome clearly call for the consideration of somatic severity of self-poisoning in guiding further psychiatric care, which was not the case in the present series, given the similar frequency of transfer to the psychiatric ward in the two groups. Indeed, in our centre, psychiatrists are advised to rely solely on psychiatric diagnoses and intensity of suicidal ideation at the time of evaluation.

The psychiatric characteristics and outcome for patients in the non-ventilated group are also noteworthy. The main diagnosis for these patients was adjustment disorders. Self-poisoning episodes in these patients are frequently in reaction to an acute disturbing life event such as loss of employment, friend or any psychosocial stressors in recovering from these events. Suicidal behaviour is more common among individuals diagnosed with adjustment disorder than individuals without this diagnosis. ${ }^{32}$ Often, these life events entailed modifications in life organisation leading to relocation, hence increasing the risk of social isolation. Indeed, in the present study, we encountered greater difficulties in reaching patients in the non-ventilated group (i.e. change of address, mobile telephone). Undoubtedly, geographical mobility and risk of social isolation are difficulties to be taken into account in order to provide effective follow-up.

Interestingly, we observed that after a certain period of time following the index episode, the vast majority of respondents were satisfied that they had been treated for their self-poisoning. This observation may be related to the ambivalence of patients who are suicidal, as intent of suicide cannot be reduced to a simplistic will to die. ${ }^{33}$ Alternatively, it may be because of the difficulty in recognising the will to die during an interview.

\section{Limitations}

Several limitations of this study should be considered. The retrospective nature of some of the data may represent a bias. However, a stable and long-standing medical team established the psychiatric records, thus ensuring consistency. With regard to the prospective aspect of the study, the number of unreachable patients was considerable and differed between the two groups. Although this in itself is a relevant result, it nonetheless limits the scope of the comparisons. A partial response to this issue was to perform an analysis restricted to pair-matched respondents. Finally, it should be emphasised that the overall rate of refusal to be interviewed was low, compared with other studies. ${ }^{25}$ The time interval between self-harm to follow-up ranged from 6 to 18 months and this may have introduced a recall decay and/or recall bias. However, as the delay between the two groups did not differ, it is our belief that this is unlikely to have had a significant impact on our results. Finally, the matching criteria could be disputed. Notably, matching on past medical history possibly selected a control group with a higher frequency of past psychiatric disorders than generally observed in unselected patients with non-severe drug overdose. In the absence of such matching, the differences between the two groups may potentially have been greater.

\section{Implications}

In conclusion, this study shows that the requirement for mechanical ventilation, taken as a single somatic severity criterion, during an episode of self-poisoning delineates a population with very frequent mood disorders at high risk of poor long-term outcome, including death. This simple criterion should be taken into account in order to adjust psychiatric care during and after the self-poisoning episode.

\footnotetext{
Elodie Baer, MD, Department of Medical Intensive Care and Hyperbaric Medicine, Angers University Hospital, Angers; Carole Barré, MD, Department of Psychiatry, Angers University Hospital, Angers; Carole Fleury, MD, Department of Medical Intensive Care and Hyperbaric Medicine, Angers University Hospital, Angers; Claire de Montchenu, MD, Department of Psychiatry, Angers University Hospital, Angers; Jean-Bernard Garré, MD, Department of Psychiatry, Angers University Hospital, Angers; Nicolas Lerolle, MD, PhD, Department of Medical Intensive Care and Hyperbaric Medicine, Angers University Hospital, Angers; Bénédicte Gohier MD, PhD, Department of Psychiatry, Angers University Hospital, Angers, France

Correspondence: Nicolas Lerolle, Département de Réanimation Médicale et de Médecine Hyperbare, Centre Hospitalier Universitaire, 4 rue Larrey, F-49 933 Angers Cedex 9, France. Email: nicolas.lerolle@univ-angers.fr

First received 9 Nov 2013, final revision 9 Dec 2014, accepted 19 Dec 2014
}

\section{Funding}

This work was supported by institutional funding: Angers University, Angers and Angers University Hospital, Angers, France. 


\section{References}

1 Borges G, Nock MK, Haro Abad JM, Hwang I, Sampson NA, Alonso J, et al. Twelve-month prevalence of and risk factors for suicide attempts in the World Health Organization World Mental Health Surveys. J Clin Psychiatry 2010; 71: 1617-28.

2 Gould MS, Greenberg T, Velting DM, Shaffer D. Youth suicide risk and preventive interventions: a review of the past 10 years. J Am Acad Child Adolesc Psychiatry 2003; 42: 386-405.

3 Denning DG, Conwell Y, King D, Cox C. Method choice, intent, and gender in completed suicide. Suicide Life Threat Behav 2000; 30: 282-8.

4 Groupement d'Etudes et de Prévention du Suicide (GEPS). Suicides, Étude et Prévention. [Suicides, Study and Prevention.] GEPS, 2008 (http://www.geps.asso.fr/articles.php?rub = 8).

5 Krug EG, Mercy JA, Dahlberg LL, Zwi AB. The world report on violence and health. Lancet 2002; 360: 1083-8.

6 Mocicki EK. Identification of suicide risk factors using epidemiologic studies. Psychiatr Clin North Am 1997; 20: 499-517.

7 Hawton K, van Heeringen K. Suicide. Lancet 2009; 373: 1372-81.

8 Bergen H, Hawton K, Waters K, Ness J, Cooper J, Steeg S, et al. Premature death after self-harm: a multicentre cohort study. Lancet 2012; $\mathbf{3 8 0}$ 1568-74.

9 Saunders KEA, Hawton K. Clinical assessment and crisis intervention for the suicidal bipolar disorder patient. Bipolar Disord 2013; 15: 575-83.

10 Carter GL, Clover K, Whyte IM, Dawson AH, D'Este C. Postcards from the EDge project: randomised controlled trial of an intervention using postcards to reduce repetition of hospital treated deliberate self poisoning. BMJ 2005 331: 805 .

11 Guthrie E, Kapur N, Mackway-Jones K, Chew-Graham C, Moorey J, Mendel E, et al. Randomised controlled trial of brief psychological intervention after deliberate self poisoning. BMJ 2001; 323: 135-8.

12 Vaiva G, Vaiva G, Ducrocq F, Meyer P, Mathieu D, Philippe A, et al. Effect of telephone contact on further suicide attempts in patients discharged from an emergency department: randomised controlled study. BMJ 2006; 332 $1241-5$

13 Harris EC, Barraclough B. Suicide as an outcome for mental disorders. A meta-analysis. Br J Psychiatry 1997; 170: 205-28.

14 Brown GK, Beck AT, Steer RA, Grisham JR. Risk factors for suicide in psychiatric outpatients: a 20-year prospective study. J Consult Clin Psychol 2000; 68: 371-7.

15 Cheng AT, Chen TH, Chen CC, Jenkins R. Psychosocial and psychiatric risk factors for suicide. Case-control psychological autopsy study. Br J Psychiatry 2000; 177: 360-5

16 Wenzel A, Berchick ER, Tenhave T, Halberstadt S, Brown GK, Beck AT. Predictors of suicide relative to other deaths in patients with suicide attempts and suicide ideation: a 30-year prospective study. J Affect Disord 2011; 132: 375-82.

17 Kapur N, Clements C, Bateman N, Foëx B, Mackway-Jones K, Hawton K, et al. Self-poisoning suicide deaths in England: could improved medical management contribute to suicide prevention? QJM 2010; 103: 765-75.
18 Le Gall JR, Lemeshow S, Saulnier F. A new Simplified Acute Physiology Score (SAPS II) based on a European/North American multicenter study. JAMA 1993; 270: 2957-63.

19 Fagon JY, Chastre J, Novara A, Medioni P, Gibert C. Characterization of intensive care unit patients using a model based on the presence or absence of organ dysfunctions and/or infection: the ODIN model. Intensive Care Med 1993: 19: 137-44.

20 Katz S. Assessing self-maintenance: activities of daily living, mobility, and instrumental activities of daily living. J Am Geriatr Soc 1983; 31: 721-7.

21 Gandek B, Ware JE, Aaronson NK, Apolone G, Bjorner JB, Brazier JE, et al. Cross-validation of item selection and scoring for the SF-12 Health Survey in nine countries: results from the IQOLA Project. International Quality of Life Assessment. J Clin Epidemiol 1998; 51: 1171-8.

22 Zigmond AS, Snaith RP. The hospital anxiety and depression scale. Acta Psychiatr Scand 1983; 67: 361-70.

23 Nordentoft M, Breum L, Munck LK, Nordestgaard AG, Hunding A, Laursen Bjaeldager PA. High mortality by natural and unnatural causes: a 10 year follow up study of patients admitted to a poisoning treatment centre after suicide attempts. BMJ 1993; 306: 1637-41.

24 Owens D, Wood C, Greenwood DC, Hughes T, Dennis M. Mortality and suicide after non-fatal self-poisoning: 16-year outcome study. Br J Psychiatry 2005; 187: 470-5.

25 Douglas J, Cooper J, Amos T, Webb R, Guthrie E, Appleby L. 'Near-fatal' deliberate self-harm: characteristics, prevention and implications for the prevention of suicide. J Affect Disord 2004; 79: 263-8.

26 Scocco $\mathrm{P}$, Marietta $\mathrm{P}$, Tonietto $\mathrm{M}$, Dello Buono $\mathrm{M}$, De Leo $\mathrm{D}$. The role of psychopathology and suicidal intention in predicting suicide risk: a longitudinal study. Psychopathology 2000; 33: 143-50.

27 Beautrais AL, Joyce PR, Mulder RT, Fergusson DM, Deavoll BJ, Nightingale SK. Prevalence and comorbidity of mental disorders in persons making serious suicide attempts: a case-control study. Am J Psychiatry 1996; 153: 1009-14.

28 Suominen $\mathrm{K}$, Isometsä E, Ostamo A, Lönnqvist J. Level of suicidal intent predicts overall mortality and suicide after attempted suicide: a 12-year follow-up study. BMC Psychiatry 2004; 4: 11.

29 Crump C, Sundquist K, Sundquist J, Winkleby MA. Sociodemographic, psychiatric and somatic risk factors for suicide: a Swedish national cohort study. Psychol Med 2014; 44: 279-89.

30 Griffiths J, Fortune G, Barber V, Young JD. The prevalence of post traumatic stress disorder in survivors of ICU treatment: a systematic review. Intensive Care Med 2007; 33: 1506-18

31 Pandharipande PP, Girard TD, Jackson JC, Morandi A, Thompson JL, Pun BT, et al. Long-term cognitive impairment after critical illness. New Engl J Med 2013; 369: 1306-16.

32 Kryzhanovskaya L, Canterbury R. Suicidal behavior in patients with adjustment disorders. Crisis 2001; 22: 125-31.

33 David AS, Hotopf M, Moran P, Owen G, Szmukler G, Richardson G. Mentally disordered or lacking capacity? Lessons for management of serious deliberate self harm. BMJ 2010; 341: c4489 